Supporting Information

\title{
Role of Polyhedron Unit in Distinct Photophysics of Zero-Dimensional Organic-Inorganic Hybrid Tin Halide Compounds
}

\author{
Xiaoyu Liu, Yuanyuan Li, Tianyuan Liang, and Jiyang Fan* \\ School of Physics, Southeast University, Nanjing 211189, P. R. China \\ *E-mail: jyfan@seu.edu.cn
}

Experimental Section. Materials. $\mathrm{SnO}$ (zinc oxide, Aladdin, 99.9\%), $\mathrm{HCl}$ (hydrochloric acid, Linfeng, 38\%), $\mathrm{HBr}$ (hydrogen bromide, Aladdin, 48.0\%), HI (hydriodic acid, Macklin, 55.0\%), $\mathrm{C}_{10} \mathrm{H}_{24} \mathrm{~N}_{4}$ (1,4,8,11-tetraazacyclotetradecane, Macklin, 98\%). All materials were used without further purification. Synthesis of $\left(\mathrm{C}_{10} \mathrm{H}_{28} \mathrm{~N}_{4} \mathrm{Cl}_{2}\right) \mathrm{SnCl}_{4} \cdot 2 \mathrm{H}_{2} \mathrm{O},\left(\mathrm{C}_{10} \mathrm{H}_{28} \mathrm{~N}_{4}\right) \mathrm{SnBr}_{6} \cdot 4 \mathrm{H}_{2} \mathrm{O}$, and $\left(\mathrm{C}_{10} \mathrm{H}_{28} \mathrm{~N}_{4}\right) \mathrm{SnI}_{6} \cdot 4 \mathrm{H}_{2} \mathrm{O}$. All the compounds were synthesized by using a similar method. For synthesis of $\left(\mathrm{C}_{10} \mathrm{H}_{28} \mathrm{~N}_{4} \mathrm{Cl}_{2}\right) \mathrm{SnCl}_{4} \cdot 2 \mathrm{H}_{2} \mathrm{O}, \mathrm{C}_{10} \mathrm{H}_{24} \mathrm{~N}_{4}(0.05 \mathrm{mmol})$ was dissolved in deionized water $(1.0 \mathrm{~mL}), \mathrm{SnO}(0.05 \mathrm{mmol})$ was dissolved in hydrochloric acid $(1.0 \mathrm{~mL})$ [HBr for $\left(\mathrm{C}_{10} \mathrm{H}_{28} \mathrm{~N}_{4}\right) \mathrm{SnBr}_{6} \cdot 4 \mathrm{H}_{2} \mathrm{O}$ and $\mathrm{HI}$ for $\left.\left(\mathrm{C}_{10} \mathrm{H}_{28} \mathrm{~N}_{4}\right) \mathrm{SnI}_{6} \cdot 4 \mathrm{H}_{2} \mathrm{O}\right]$. Subsequently, the two solutions were quickly mixed and reacted at room temperature for $12 \mathrm{~h}$. Then the transparent crystals were produced in the solution. The synthesized crystals can remain stable in the ambient atmosphere for over a month; but they are unstable in the original solution and no luminescence can be observed after half a month. The concentrations of the reactants affect the sizes of the products, which can be controlled by controlling the amount of water.

Characterization. The UV-Vis absorption spectra were recorded by using a UVVis spectrophotometer (HITACHI U-3900). The photoluminescence (PL) spectra, PL excitation spectra, and time-resolved PL spectra were measured at room temperature by using a fluorolog3-TCSPC spectrofluorometer (HORIBA JOBIN YVON) with a Xe lamp and pulsed-laser diodes as light sources. The Raman measurement was performed at room temperature on a Jobin Yvon Lab RAM HR800 micro-Raman spectrometer with a $532 \mathrm{~nm}$ laser line. The powder X-ray diffraction (XRD) was performed on an X-ray powder diffractometer [Smartlab (3)]. The single crystal X-ray diffraction data were collected at $193 \mathrm{~K}$ from an X'Pert PRO MPD system equipped with the Mo Ka source. The crystal structures were resolved and refined by using SHELXS and SHELXL in conjunction with the Olex2 package. ${ }^{1,2}$

Computational methods. The density function theory (DFT) calculations were performed by using the Vienna ab initio simulation package (VASP) $5.4 .4 .^{3}$ The projected augmented wave (PAW) method was used to describe the interaction between the ionic core and the valence electrons. ${ }^{4}$ All the crystal structures obtained from the single crystal XRD were optimized using the Perdew-Burke-Ernzerhof (PBE) exchange-correlation functional. ${ }^{5}$ The lattice constants were relaxed and the atomic positions were rearranged until the force on each atom was smaller than 0.005 meV/Angstrom. The kinetic energy cutoff of the plane-wave basis was $500 \mathrm{eV}$. The Monkhorst-Pack k-mesh took $3 \times 3 \times 3$ for $\left(\mathrm{C}_{10} \mathrm{H}_{28} \mathrm{~N}_{4} \mathrm{Cl}_{2}\right) \mathrm{SnCl}_{4} \cdot 2 \mathrm{H}_{2} \mathrm{O}, 5 \times 4 \times 2$ for $\left(\mathrm{C}_{10} \mathrm{H}_{28} \mathrm{~N}_{4}\right) \mathrm{SnBr}_{6} \cdot 4 \mathrm{H}_{2} \mathrm{O}$, and $4 \times 4 \times 4$ for $\left(\mathrm{C}_{10} \mathrm{H}_{28} \mathrm{~N}_{4}\right) \mathrm{SnI}_{6} \cdot 4 \mathrm{H}_{2} \mathrm{O}$. The electronic structure and density of states (DOS) of the three compounds were calculated by using 
the hybrid PBE0 exchange-correlation functional. ${ }^{6}$ The total energy of the excited state was calculated in the case of raising an electron in the valence band into the conduction band. The visualization and analysis of the crystal structures and isosurfaces of HOMO and LUMO were performed by using VESTA. ${ }^{7}$ The dynamical matrix obtained from VASP was used in Phonopy ${ }^{8}$ to evaluate the phonon frequencies. The density functional perturbation theory was used to predict the phonon energies at a $2 \times 2 \times 2$ grid. The phonon DOS was interpolated from this grid using the interatomic force constants.

Table S1. Crystal data and structure refinement for $\left(\mathrm{C}_{10} \mathrm{H}_{28} \mathrm{~N}_{4} \mathrm{Cl}_{2}\right) \mathrm{SnCl}_{4} \cdot 2 \mathrm{H}_{2} \mathrm{O}$.

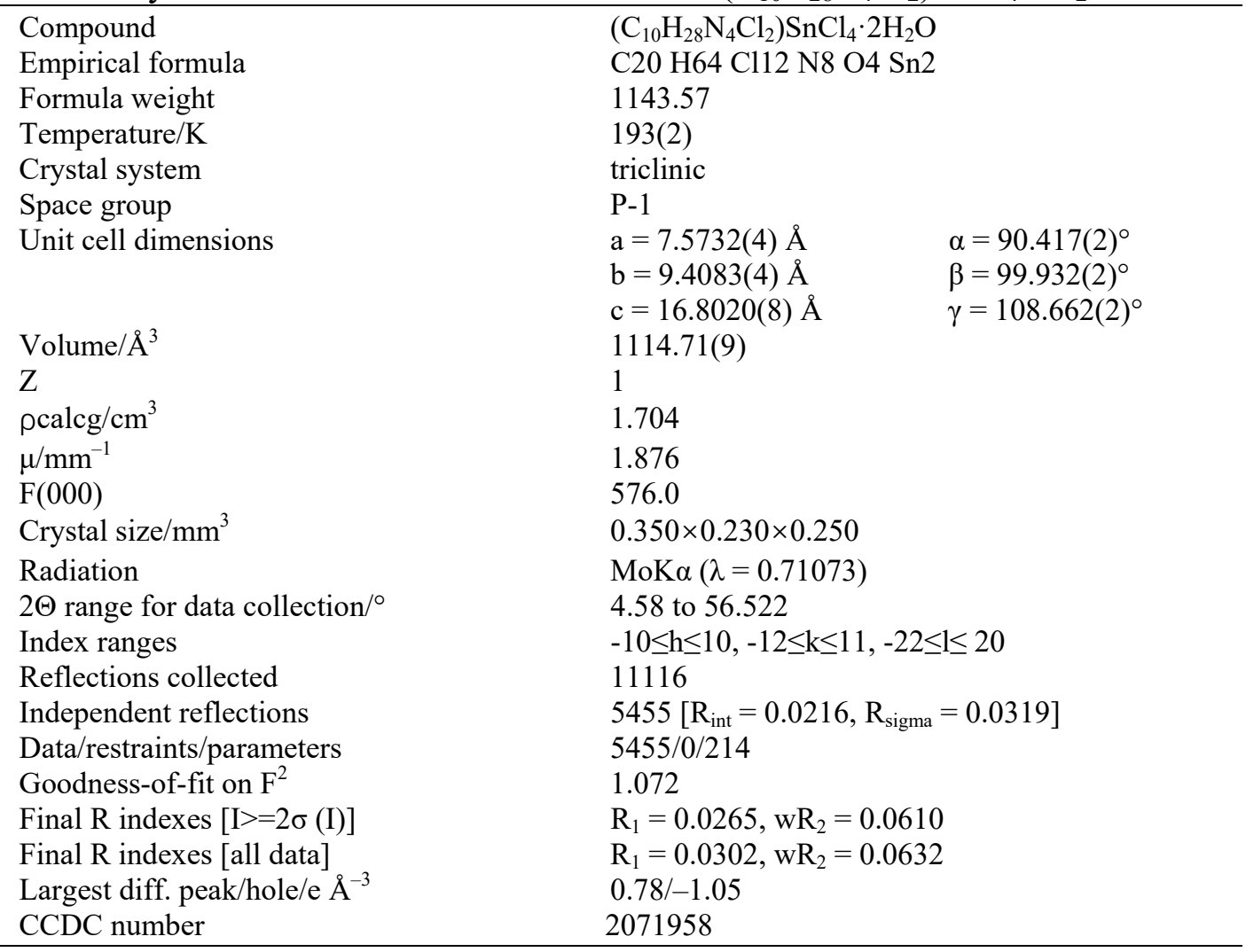


Table S2. Crystal data and structure refinement for $\left(\mathrm{C}_{10} \mathrm{H}_{28} \mathrm{~N}_{4}\right) \mathrm{SnBr}_{6} \cdot 4 \mathrm{H}_{2} \mathrm{O}$.

Compound

Empirical formula

Formula weight

Temperature/K

Crystal system

Space group

Unit cell dimensions

Volume $/ \AA^{3}$

$\mathrm{Z}$

pcalcg $/ \mathrm{cm}^{3}$

$\mu / \mathrm{mm}^{-1}$

$\mathrm{F}(000)$

Crystal size $/ \mathrm{mm}^{3}$

Radiation

$2 \Theta$ range for data collection ${ }^{\circ}$

Index ranges

Reflections collected

Independent reflections

Data/restraints/parameters

Goodness-of-fit on $\mathrm{F}^{2}$

Final $\mathrm{R}$ indexes $[\mathrm{I}>=2 \sigma(\mathrm{I})]$

Final $\mathrm{R}$ indexes [all data]

Largest diff. peak/hole/e $\AA^{-3}$

CCDC number
$\left(\mathrm{C}_{10} \mathrm{H}_{28} \mathrm{~N}_{4}\right) \mathrm{SnBr}_{6} \cdot 4 \mathrm{H}_{2} \mathrm{O}$

C10 H36 Br6 N4 O4 Sn

874.58

193(2)

triclinic

$\mathrm{P}-1$

$\mathrm{a}=8.0987(5) \AA$

$\alpha=109.177(2)^{\circ}$

$\mathrm{b}=9.2747(5) \AA$

$\beta=112.240(2)^{\circ}$

$\mathrm{c}=10.1512(7) \AA$

$\gamma=97.800(2)^{\circ}$

636.43(7)

1

2.282

10.442

416.0

$0.550 \times 0.230 \times 0.410$

$\operatorname{MoK} \alpha(\lambda=0.71073)$

4.764 to 56.618

$-10 \leqslant \mathrm{~h} \leqslant 10,-12 \leqslant \mathrm{k} \leqslant 12,-13 \leqslant 1 \leqslant 10$

6307

$3117\left[\mathrm{R}_{\mathrm{int}}=0.0274, \mathrm{R}_{\mathrm{sigma}}=0.0410\right]$

$3117 / 0 / 121$

1.068

$\mathrm{R}_{1}=0.0300, \mathrm{wR}_{2}=0.0845$

$\mathrm{R}_{1}=0.0340, \mathrm{wR}_{2}=0.0874$

$0.98 /-2.29$

2071964 
Table S3. Crystal data and structure refinement for $\left(\mathrm{C}_{10} \mathrm{H}_{28} \mathrm{~N}_{4}\right) \mathrm{SnI}_{6} \cdot 4 \mathrm{H}_{2} \mathrm{O}$.

\section{Compound}

Empirical formula

Formula weight

Temperature/K

Crystal system

Space group

Unit cell dimensions

Volume $/ \AA^{3}$

$\mathrm{Z}$

pcalcg $/ \mathrm{cm}^{3}$

$\mu / \mathrm{mm}^{-1}$

$\mathrm{F}(000)$

Crystal size $/ \mathrm{mm}^{3}$

Radiation

$2 \Theta$ range for data collection/ ${ }^{\circ}$ Index ranges

Reflections collected

Independent reflections

Data/restraints/parameters

Goodness-of-fit on $\mathrm{F}^{2}$

Final $\mathrm{R}$ indexes $[\mathrm{I}>=2 \sigma(\mathrm{I})]$

Final $\mathrm{R}$ indexes [all data]

Largest diff. peak/hole/e $\AA^{-3}$

CCDC number
$\left(\mathrm{C}_{10} \mathrm{H}_{28} \mathrm{~N}_{4}\right) \mathrm{SnBr}_{6} \cdot 4 \mathrm{H}_{2} \mathrm{O}$

C10 H36 I6 N4 O4 Sn

1156.52

193(2)

triclinic

P-1

$a=8.5749(4) \AA$

$\alpha=64.487(2)^{\circ}$

$\mathrm{b}=9.6438(6) \AA$

$\beta=66.7210(10)^{\circ}$

$\mathrm{c}=10.5359(5) \AA$

$\gamma=80.955(2)^{\circ}$

$722.20(7)$

2.659

7.316

524.0

$0.210 \times 0.230 \times 0.180$

$\operatorname{MoK} \alpha(\lambda=0.71073)$

4.606 to 56.57

$-11 \leqslant \mathrm{~h} \leqslant 11,-10 \leqslant \mathrm{k} \leqslant 12,-13 \leqslant 1 \leqslant 14$

7212

$3536\left[\mathrm{R}_{\mathrm{int}}=0.0206, \mathrm{R}_{\mathrm{sigma}}=0.0307\right]$

$3536 / 0 / 121$

1.039

$\mathrm{R}_{1}=0.0211, \mathrm{wR}_{2}=0.0456$

$\mathrm{R}_{1}=0.0251, \mathrm{wR}_{2}=0.0478$

$0.76 /-0.91$

2072001 
Table S4. Electron distribution and coordinates of the different ions in the supercell of the $\left(\mathrm{C}_{10} \mathrm{H}_{28} \mathrm{~N}_{4} \mathrm{Cl}_{2}\right) \mathrm{SnCl}_{4} \cdot 2 \mathrm{H}_{2} \mathrm{O}$ single crystal corresponding to the ground state.

\begin{tabular}{|c|c|c|c|c|c|}
\hline \multirow[b]{2}{*}{ Sn } & \multicolumn{2}{|c|}{$x$} & \multirow{2}{*}{$\begin{array}{l}Y \\
6.644788\end{array}$} & \multirow{2}{*}{$Z_{12.97995}$} & \multirow{2}{*}{$\begin{array}{l}\text { CHARGE } \\
12.79237\end{array}$} \\
\hline & 1 & -2.81867 & & & \\
\hline & 2 & 4.733038 & 1.544652 & 4.022632 & 12.79237 \\
\hline $\mathrm{Cl}$ & 3 & -2.99533 & 5.629003 & 15.73905 & 7.738308 \\
\hline & 4 & 4.909695 & 2.560437 & 1263537 & 7.738304 \\
\hline & 5 & 3.382342 & 4.70205 & 12.29796 & 7.681084 \\
\hline & 6 & -1.46798 & 3.48739 & 4.704623 & 7681084 \\
\hline & 7 & -4.78569 & 8.105641 & 13.66166 & 7.668392 \\
\hline & 8 & 6.700059 & 0.083799 & 3.340921 & 7.668392 \\
\hline & 9 & -3.19042 & 7.512625 & 10.34269 & 7.724342 \\
\hline & 10 & 5.104785 & 0.676815 & 6.659892 & 7.724347 \\
\hline & 11 & 2310673 & 0.568843 & 13.65089 & 7.740687 \\
\hline & 12 & -0.39631 & 7.620597 & 3.351691 & 7.740687 \\
\hline & 13 & -0.16274 & 4.317381 & 11.32022 & 7.744551 \\
\hline & 14 & 2.077103 & 3.872059 & 5.682364 & 7.744551 \\
\hline 0 & 15 & 3.124103 & -0.71041 & 16.36735 & 7.283707 \\
\hline & 16 & -1.20974 & 8.899848 & 0.635231 & 7.283701 \\
\hline & 17 & 4.523405 & 4.883653 & 7.246345 & 7.299289 \\
\hline & 18 & -2.60904 & 3.305787 & 9.756238 & 7.299333 \\
\hline H & 19 & 5.433597 & 0.687929 & 0.085484 & 0373839 \\
\hline & 20 & -3.51923 & 7.501511 & 169171 & 0.373839 \\
\hline & 21 & 2.829458 & -0.32573 & 15.50224 & 037575 \\
\hline & 22 & -0.91509 & 8.515173 & 1.50034 & 037575 \\
\hline & 23 & 1.136887 & 7.004073 & 16.35917 & 044699 \\
\hline & 24 & 0.777478 & 1.185367 & 0.643418 & 044699 \\
\hline & 25 & 2.57944 & 6.112 .71 & 16.09844 & 0485668 \\
\hline & 26 & -0.66508 & 2.077269 & 0.90414 & 0485668 \\
\hline & 27 & 1.157844 & 2.176494 & 13.91539 & 0.483471 \\
\hline & 28 & 0.756521 & 6.012946 & 3.087197 & 0483471 \\
\hline & 29 & 0.553323 & 3.56708 & 13.15797 & 0492967 \\
\hline & 30 & 1.361045 & 4.622351 & 3.844614 & 0492967 \\
\hline & 31 & -144443 & 6.874927 & 5.094741 & 0.532079 \\
\hline & 32 & 3.358796 & 1314513 & 11.90784 & 0.532079 \\
\hline & 33 & -2.1489 & 5.959691 & 6.387933 & 0.4594 \\
\hline & 34 & 4.06326 & 2.229749 & 10.61465 & 04594 \\
\hline & 35 & 1.007888 & 5.884618 & 10.64452 & 0.475093 \\
\hline & 36 & 0.906477 & 2.304822 & 6.35806 & 0.475093 \\
\hline & 37 & 2.42067 & 6.822225 & 10.44024 & 045942 \\
\hline & 38 & -0.50631 & 1367215 & 6.562348 & 0.45942 \\
\hline & 39 & -1.17996 & 2.094749 & 13.6705 & 0897409 \\
\hline & 40 & 3.094329 & 6.094691 & 3.332084 & 0.897409 \\
\hline & 41 & -138724 & 3.657453 & 14.49051 & 0927396 \\
\hline & 42 & 3.301607 & 4. 531987 & 2.512074 & 0.927396 \\
\hline & 43 & 2.440427 & 3.920777 & 14.63618 & 0.84799 \\
\hline & 44 & -0.52606 & 4.268663 & 2.366399 & 0.84799 \\
\hline & 45 & 1.473656 & 3.322 .139 & 15.99737 & 0910418 \\
\hline & 46 & 0.440709 & 4.867301 & 1.005212 & 0.910418 \\
\hline & 47 & -2.54016 & 7903421 & 7.663786 & 0.880064 \\
\hline & 48 & 4.454524 & 0.286019 & 9.338797 & 0890064 \\
\hline & 49 & -3.3896 & 7.873641 & 6.111948 & 0871905 \\
\hline & 50 & 5.303961 & 0.315799 & 10.89064 & 0871905 \\
\hline & 51 & 0.405812 & 7.525911 & 6.329359 & 0890134 \\
\hline & 52 & 1.508553 & 0.663529 & 10.67322 & 0.890134 \\
\hline & 53 & 0.221948 & 5.764951 & 6.266274 & 0.885159 \\
\hline & 54 & 1692417 & 2.424489 & 10.73631 & 0.885159 \\
\hline & 55 & 1.061911 & 5.88122 & 14.31355 & 0883973 \\
\hline & 56 & 0.852454 & 2.30822 & 2.689033 & 0.883973 \\
\hline & 57 & -0.20216 & 5315111 & 15.42669 & 0902579 \\
\hline & 58 & 2.116521 & 2.874329 & 1.575893 & 0902579 \\
\hline & 59 & -0.31441 & 2.57541 & 16.5751 & 0.93363 \\
\hline & 50 & 2.228778 & 5.61403 & 0.427482 & 0.933496 \\
\hline & 51 & -0.38786 & 1.022744 & 15.74589 & 0.948008 \\
\hline & 52 & 2302231 & 7.166696 & 1.256694 & 0.948009 \\
\hline & 53 & 4.784431 & 7.021619 & 1.397728 & 0.881425 \\
\hline & 64 & -2.87007 & 1.167821 & 15.60486 & 0.881425 \\
\hline & 65 & 4.759281 & 5.362853 & 0.759354 & 0.921614 \\
\hline & 56 & -284491 & 2.826578 & 16.24323 & 0.921613 \\
\hline & 57 & 1.818997 & 5.742 .45 & 8.437559 & 0913485 \\
\hline & 58 & 0.095368 & 2447295 & 8565024 & 0913483 \\
\hline & 69 & 1831779 & 7.504372 & 8.27404 & 0905652 \\
\hline & 70 & 0.082583 & 0.685077 & 8.728543 & 0.905652 \\
\hline & 71 & 0.819976 & 7.775666 & 11.95374 & 0900127 \\
\hline & 72 & 1.094389 & 0.413774 & 5.048844 & 0.900127 \\
\hline & 73 & -0.37397 & 7.832636 & 10.64147 & 0.900535 \\
\hline & 74 & 2.288337 & 0.356304 & 6361109 & \\
\hline & 75 & 5.020318 & 4.048296 & 7.349822 & 0358049 \\
\hline & 76 & -3.10595 & 4.141 .44 & 9.652761 & 0358049 \\
\hline & 77 & 3.741265 & 4.611485 & 6.691181 & 0352267 \\
\hline & 78 & -1.8269 & 3.577955 & 10.3114 & 0.352267 \\
\hline & 79 & -0.71016 & 7.486178 & 8.641864 & 0.972928 \\
\hline & 30 & 2.624523 & 0.703262 & 8360719 & 0972719 \\
\hline & 31 & -0.67761 & 5.733041 & 8.605632 & \\
\hline & 32 & 2.59197 & 2.456399 & 8.396951 & 0.913 \\
\hline
\end{tabular}

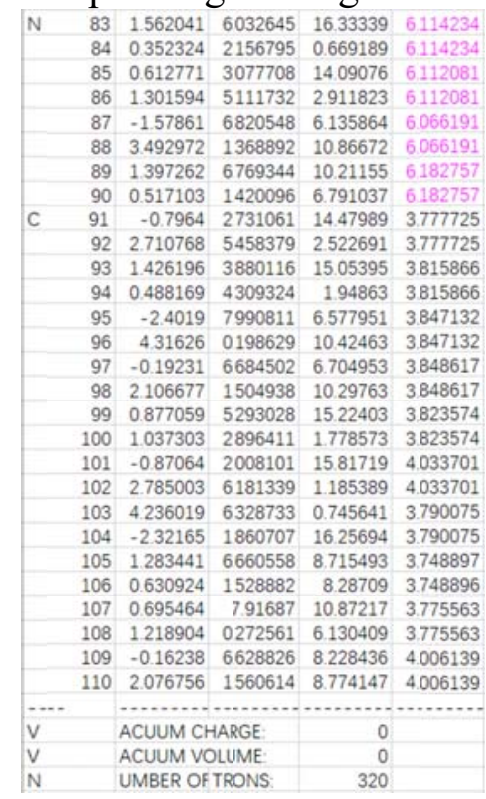


Table S5. Electron distribution and coordinates of the different ions in the supercell of the $\left(\mathrm{C}_{10} \mathrm{H}_{28} \mathrm{~N}_{4}\right) \mathrm{SnBr}_{6} \cdot 4 \mathrm{H}_{2} \mathrm{O}$ single crystal corresponding to the ground state.

\begin{tabular}{|c|c|c|c|c|c|}
\hline \multicolumn{3}{|r|}{$\mathrm{X}$} & \multirow{2}{*}{$\begin{array}{l}Y \\
4.711693\end{array}$} & \multirow{2}{*}{$\begin{array}{l}Z^{2} \\
0.003537\end{array}$} & \multirow{2}{*}{$\begin{array}{l}\text { CHARGE } \\
12.86721\end{array}$} \\
\hline Sn & 1 & 3.530332 & & & \\
\hline \multirow[t]{6}{*}{$\mathrm{Br}$} & 2 & 3.895396 & 6.669874 & 2.270295 & 690317 \\
\hline & 3 & -0.71029 & -1.29855 & 6.374218 & 7.690313 \\
\hline & 4 & 2.139012 & 1.957192 & 7.670651 & 7.667616 \\
\hline & 5 & 1.046095 & 3.41413 & 0.973862 & 7.667606 \\
\hline & 6 & -1.99568 & 2.525564 & 6.857246 & 7.688022 \\
\hline & 7 & 5.180782 & $2.84575 B$ & 1.787267 & 7.688028 \\
\hline \multirow[t]{4}{*}{ N } & 8 & 4.049892 & -0.35099 & 6.412944 & 6.094334 \\
\hline & 9 & -0.86479 & 5.722312 & 2.231569 & 6.094334 \\
\hline & 10 & 1.209454 & 0.109409 & 2.307654 & 6.078548 \\
\hline & 11 & 1.975653 & 5.261913 & 6.336859 & 6.078548 \\
\hline \multirow[t]{36}{*}{$\mathrm{H}$} & 12 & 3.593332 & 0.442544 & 6.943963 & 0.50605 \\
\hline & 13 & -0.40823 & 4.928778 & 1.70055 & 0.50605 \\
\hline & 14 & -3.20601 & -0.46731 & 6.644762 & 0.499985 \\
\hline & 15 & 6.39112 & 5.838627 & 1.999751 & 0.499985 \\
\hline & 16 & -1.42093 & -3.13786 & 7.8 & 0.384442 \\
\hline & 17 & 4.60604 & 8.509181 & 0.7 & 384442 \\
\hline & 18 & -2.66615 & & & 0.352465 \\
\hline & 19 & 5.85 & 0.7 & & 2465 \\
\hline & 20 & & & & 0.322343 \\
\hline & 21 & 4.09 & 3.7 & & 2343 \\
\hline & 22 & -1.35466 & 2.05 & 3.1 & 0.343958 \\
\hline & 23 & 4.53 & 3.3 & & 0.343958 \\
\hline & 24 & 0.622763 & 0.88 & & 4855 \\
\hline & 25 & 2.56 & 4.48 & & 0.474855 \\
\hline & 26 & 1.33 & 0.2 & & 4781 \\
\hline & 27 & 1.85 & 5.116693 & & 0.474781 \\
\hline & 28 & -0.5 & -0 & & 2186 \\
\hline & 29 & & & & 2186 \\
\hline & 30 & 0.3 & -1 & & 776 \\
\hline & 31 & & & & 0.889776 \\
\hline & 32 & -0.3 & 7.0 & & 244 \\
\hline & 33 & & & & 0.816244 \\
\hline & 34 & $0.8 \mathrm{~s}$ & 6. & & 345 \\
\hline & 35 & 2.285243 & -1.48122 & 6.64 & 0.932345 \\
\hline & 36 & 2.99 & 1.1 & & 552 \\
\hline & 37 & 0.188216 & 4.208645 & 6.0 & 0.885552 \\
\hline & 38 & 3.200874 & -0.59429 & 2.4 & 5522 \\
\hline & 39 & -0.01577 & 5.9 & 6.2 & 0.899522 \\
\hline & 40 & 4.607846 & -0.7 & 4.40 & 0.878851 \\
\hline & 41 & -1.42274 & 6.12 & 4.2 & 0.878851 \\
\hline & 42 & 4.519165 & 083 & 1063 & 0.8617 \\
\hline & 43 & -1.33406 & 4.409247 & 41 & 0.8617 \\
\hline & 44 & & & & \\
\hline & 45 & 1.155361 & 6.205307 & 3.93 & 0.971917 \\
\hline & 46 & & & & \\
\hline & 47 & 1.125073 & 4.449341 & 3.768367 & 0.891218 \\
\hline \multirow[t]{4}{*}{ O } & 48 & & & & \\
\hline & 49 & 6.277237 & -0.06223 & 0.469086 & 7.306492 \\
\hline & 50 & -0.58355 & 1.744244 & 3.716519 & 7.370988 \\
\hline & 51 & 3.76866 & 3.62 & 4.9 & 7.370988 \\
\hline \multirow[t]{10}{*}{ C } & 52 & 0.420502 & -1.13963 & 2.554841 & 3.80099 \\
\hline & 53 & 2.764605 & 6.510953 & 6.089672 & 3.80099 \\
\hline & 54 & -0.17245 & 6.980803 & 1.801675 & 3.846163 \\
\hline & 55 & 3.357557 & -1.60948 & 6.842838 & 3.846163 \\
\hline & 56 & 2.589026 & 0.19444 & 2.898515 & 3.779779 \\
\hline & 57 & 0.596081 & 5.176882 & 5.745998 & 3.779779 \\
\hline & 58 & 4.009381 & -0.00631 & 4.94844 & 3.868042 \\
\hline & 59 & -0.82427 & 5.377631 & 3.696073 & 3.868042 \\
\hline & 60 & 2.581601 & 0.070376 & 4.415176 & 4.096991 \\
\hline & 61 & 0.60351 & 5.30095 & 4.229329 & 4.096991 \\
\hline & & \multicolumn{2}{|c|}{ - nen } & ................ & \\
\hline V & & \multicolumn{2}{|c|}{ ACUUM CHARGE: } & 0 & \\
\hline V & & \multicolumn{2}{|c|}{ ACUUM VOLUME: } & 0 & \\
\hline 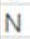 & & \multicolumn{2}{|c|}{ UMBER OF TRONS: } & 176 & \\
\hline
\end{tabular}


Table S6. Electron distribution and coordinates of the different ions in the supercell of the $\left(\mathrm{C}_{10} \mathrm{H}_{28} \mathrm{~N}_{4}\right) \mathrm{SnI}_{6} \cdot 4 \mathrm{H}_{2} \mathrm{O}$ single crystal corresponding to the ground state.

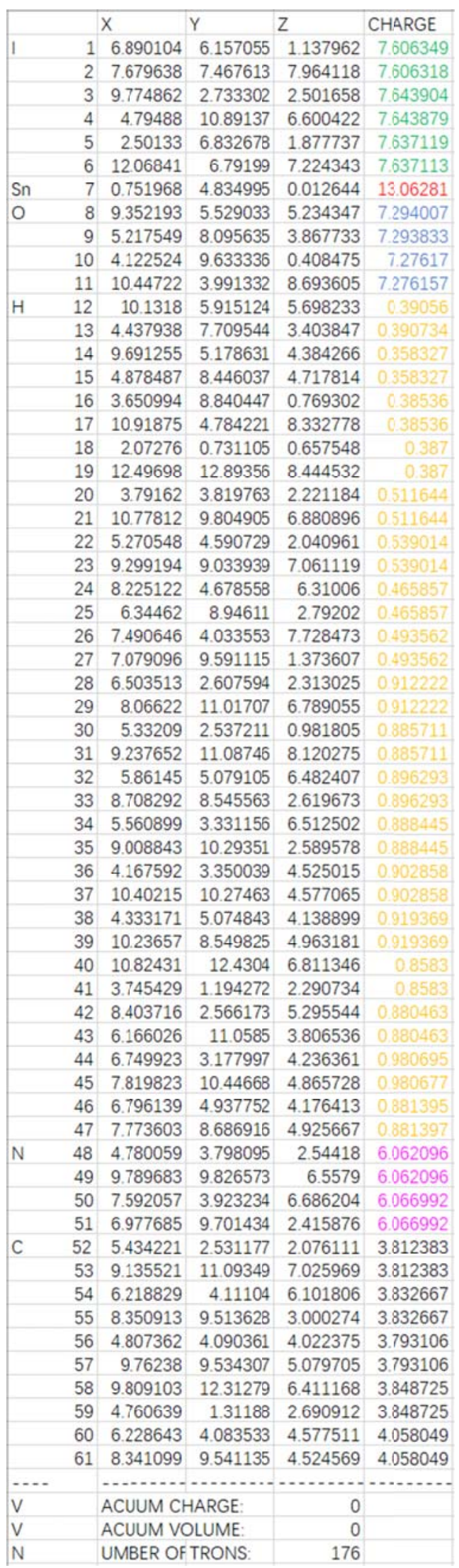



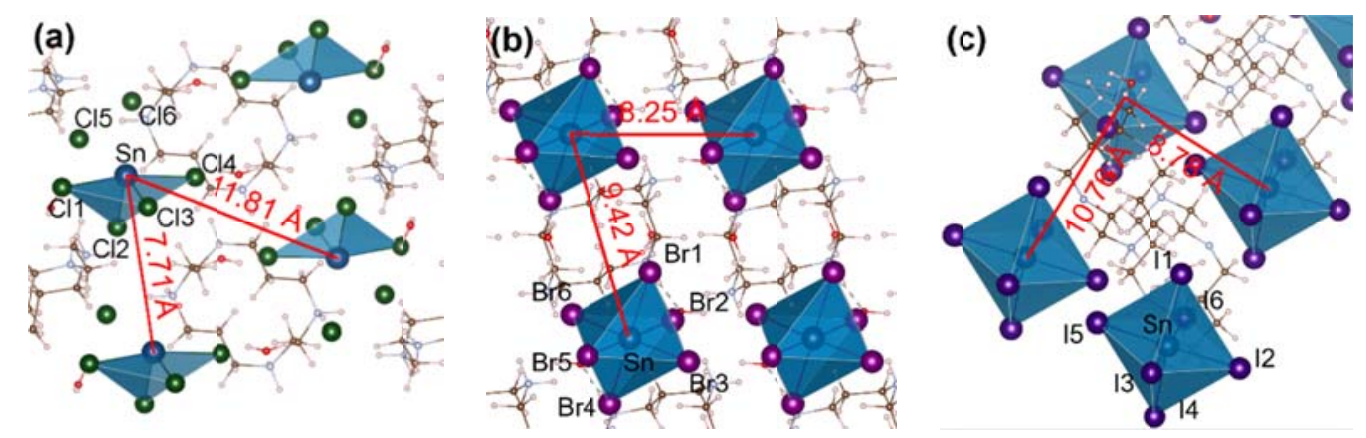

Figure S1. Crystal structures of (a) $\left(\mathrm{C}_{10} \mathrm{H}_{28} \mathrm{~N}_{4} \mathrm{Cl}_{2}\right) \mathrm{SnCl}_{4} \cdot 2 \mathrm{H}_{2} \mathrm{O}$, $\left(\mathrm{C}_{10} \mathrm{H}_{28} \mathrm{~N}_{4}\right) \mathrm{SnBr}_{6} \cdot 4 \mathrm{H}_{2} \mathrm{O}$, and (c) $\left(\mathrm{C}_{10} \mathrm{H}_{28} \mathrm{~N}_{4}\right) \mathrm{SnI}_{6} \cdot 4 \mathrm{H}_{2} \mathrm{O}$.
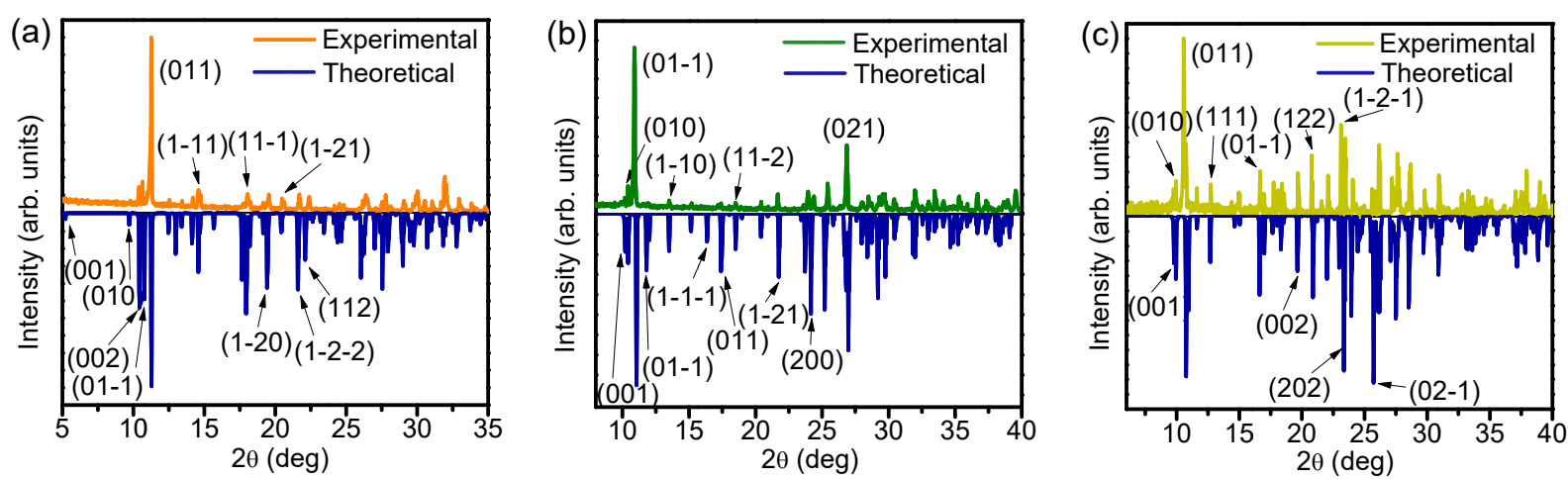

Figure S2. Comparison of measured and calculated XRD patterns of (a) $\left(\mathrm{C}_{10} \mathrm{H}_{28} \mathrm{~N}_{4} \mathrm{Cl}_{2}\right) \mathrm{SnCl}_{4} \cdot 2 \mathrm{H}_{2} \mathrm{O}$, (b) $\left(\mathrm{C}_{10} \mathrm{H}_{28} \mathrm{~N}_{4}\right) \mathrm{SnBr}_{6} \cdot 4 \mathrm{H}_{2} \mathrm{O}$, and (c) $\left(\mathrm{C}_{10} \mathrm{H}_{28} \mathrm{~N}_{4}\right) \mathrm{SnI}_{6} \cdot 4 \mathrm{H}_{2} \mathrm{O}$ powders.

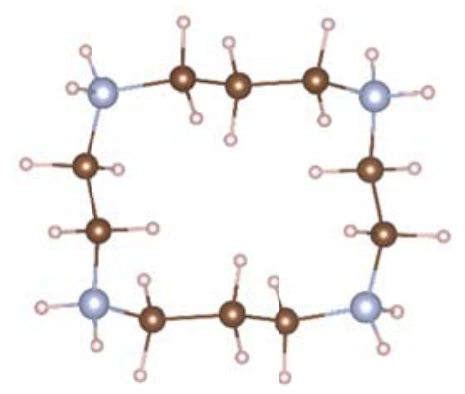

Figure S3. Chemical structure of the organic cation $\left[\mathrm{C}_{10} \mathrm{H}_{28} \mathrm{~N}_{4}\right]^{4+}$. The four secondary amines in 1,4,8,11-tetraazacyclotetradecane combine with the protons to form $\left[\mathrm{C}_{10} \mathrm{H}_{28} \mathrm{~N}_{4}\right]^{4+}$ in the acidic solution. The gray, silver, and pink balls represent $\mathrm{C}, \mathrm{N}$, and $\mathrm{H}$ atoms, respectively. 
Table S7. Distance between the $\mathrm{Sn}$ atom and the nearby $\mathrm{X}(\mathrm{X}=\mathrm{Cl}, \mathrm{Br}, \mathrm{I})$ atoms in the supercell of $\left(\mathrm{C}_{10} \mathrm{H}_{28} \mathrm{~N}_{4} \mathrm{Cl}_{2}\right) \mathrm{SnCl}_{4} \cdot 2 \mathrm{H}_{2} \mathrm{O}, \quad\left(\mathrm{C}_{10} \mathrm{H}_{28} \mathrm{~N}_{4}\right) \mathrm{SnBr}_{6} \cdot 4 \mathrm{H}_{2} \mathrm{O}$, and $\left(\mathrm{C}_{10} \mathrm{H}_{28} \mathrm{~N}_{4}\right) \mathrm{SnI}_{6} \cdot 4 \mathrm{H}_{2} \mathrm{O}$.

\begin{tabular}{cc|cc|cc}
\hline \multicolumn{2}{c|}{$\left(\mathrm{C}_{10} \mathrm{H}_{28} \mathrm{~N}_{4} \mathrm{Cl}_{2}\right) \mathrm{SnCl}_{4} \cdot 2 \mathrm{H}_{2} \mathrm{O}$} & \multicolumn{2}{c|}{$\left(\mathrm{C}_{10} \mathrm{H}_{28} \mathrm{~N}_{4}\right) \mathrm{SnBr}_{6} \cdot 4 \mathrm{H}_{2} \mathrm{O}$} & \multicolumn{2}{c}{$\left(\mathrm{C}_{10} \mathrm{H}_{28} \mathrm{~N}_{4}\right) \mathrm{SnI}_{6} \cdot 4 \mathrm{H}_{2} \mathrm{O}$} \\
\hline Label & Distance $(\AA)$ & Label & Distance $(\AA)$ & Label & Distance $(\AA)$ \\
\hline $\mathrm{Sn}-\mathrm{Cl1}$ & 2.81 & $\mathrm{Sn}-\mathrm{Br} 1$ & 3.02 & $\mathrm{Sn}-\mathrm{I} 1$ & 3.21 \\
$\mathrm{Sn}-\mathrm{Cl} 2$ & 2.56 & $\mathrm{Sn}-\mathrm{Br} 2$ & 2.97 & $\mathrm{Sn}-\mathrm{I} 2$ & 3.16 \\
$\mathrm{Sn}-\mathrm{Cl} 3$ & 2.54 & $\mathrm{Sn}-\mathrm{Br} 3$ & 3.06 & $\mathrm{Sn}-\mathrm{I} 3$ & 3.24 \\
$\mathrm{Sn}-\mathrm{Cl} 4$ & 2.95 & $\mathrm{Sn}-\mathrm{Br} 4$ & 3.02 & $\mathrm{Sn}-\mathrm{I} 4$ & 3.21 \\
$\mathrm{Sn}-\mathrm{Cl} 5$ & 3.91 & $\mathrm{Sn}-\mathrm{Br} 5$ & 2.97 & $\mathrm{Sn}-\mathrm{I} 5$ & 3.16 \\
$\mathrm{Sn}-\mathrm{Cl} 6$ & 3.66 & $\mathrm{Sn}-\mathrm{Br} 6$ & 3.06 & $\mathrm{Sn}-\mathrm{I} 6$ & 3.24 \\
\hline
\end{tabular}

Table S8. Angles of $\mathrm{X}-\mathrm{Sn}-\mathrm{X} \quad(\mathrm{X}=\mathrm{Cl}, \mathrm{Br}, \mathrm{I})$ in the supercell of $\left(\mathrm{C}_{10} \mathrm{H}_{28} \mathrm{~N}_{4} \mathrm{Cl}_{2}\right) \mathrm{SnCl}_{4} \cdot 2 \mathrm{H}_{2} \mathrm{O},\left(\mathrm{C}_{10} \mathrm{H}_{28} \mathrm{~N}_{4}\right) \mathrm{SnBr}_{6} \cdot 4 \mathrm{H}_{2} \mathrm{O}$, and $\left(\mathrm{C}_{10} \mathrm{H}_{28} \mathrm{~N}_{4}\right) \mathrm{SnI}_{6} \cdot 4 \mathrm{H}_{2} \mathrm{O}$.

\begin{tabular}{cc|cc|cc}
\hline \multicolumn{2}{c|}{$\left(\mathrm{C}_{10} \mathrm{H}_{28} \mathrm{~N}_{4} \mathrm{Cl}_{2}\right) \mathrm{SnCl}_{4} \cdot 2 \mathrm{H}_{2} \mathrm{O}$} & \multicolumn{2}{|c|}{$\left(\mathrm{C}_{10} \mathrm{H}_{28} \mathrm{~N}_{4}\right) \mathrm{SnBr}_{6} \cdot 4 \mathrm{H}_{2} \mathrm{O}$} & \multicolumn{2}{c}{$\left(\mathrm{C}_{10} \mathrm{H}_{28} \mathrm{~N}_{4}\right) \mathrm{SnI}_{6} \cdot 4 \mathrm{H}_{2} \mathrm{O}$} \\
\hline Label & Angle $\left(^{\circ}\right)$ & Label & Angle $\left({ }^{\circ}\right)$ & Label & Angle $\left(^{\circ}\right)$ \\
\hline $\mathrm{Cl1}-\mathrm{Sn}-\mathrm{Cl} 1$ & 168.76 & $\mathrm{Br} 1-\mathrm{Sn}-\mathrm{Br} 2$ & 81.98 & I1-Sn-I2 & 94.55 \\
$\mathrm{C} 12-\mathrm{Sn}-\mathrm{Cl} 3$ & 93.13 & $\mathrm{Br} 1-\mathrm{Sn}-\mathrm{Br} 3$ & 83.84 & I1-Sn-I3 & 94.72 \\
$\mathrm{C} 11-\mathrm{Sn}-\mathrm{Cl} 3$ & 84.30 & $\mathrm{Br} 1-\mathrm{Sn}-\mathrm{Br} 5$ & 98.02 & I1-Sn-I5 & 85.45 \\
$\mathrm{C} 11-\mathrm{Sn}-\mathrm{Cl} 2$ & 87.32 & $\mathrm{Br} 1-\mathrm{Sn}-\mathrm{Br} 6$ & 96.16 & I1-Sn-I6 & 85.28 \\
$\mathrm{C} 14-\mathrm{Sn}-\mathrm{Cl} 2$ & 84.57 & $\mathrm{Br} 2-\mathrm{Sn}-\mathrm{Br} 3$ & 90.33 & I2-Sn-I3 & 90.59 \\
$\mathrm{C} 14-\mathrm{Sn}-\mathrm{Cl} 3$ & 88.38 & $\mathrm{Br} 3-\mathrm{Sn}-\mathrm{Br} 5$ & 89.67 & I3-Sn-I5 & 89.41 \\
\hline
\end{tabular}

Table S9. Crystal data and structure refinement for $\left(\mathrm{C}_{10} \mathrm{H}_{28} \mathrm{~N}_{4} \mathrm{Cl}_{2}\right) \mathrm{SnCl}_{6}$ [oxidation product of $\left(\mathrm{C}_{10} \mathrm{H}_{28} \mathrm{~N}_{4} \mathrm{Cl}_{2}\right) \mathrm{SnCl}_{4} \cdot 2 \mathrm{H}_{2} \mathrm{O}$ after its storage in the original solution for half a month].

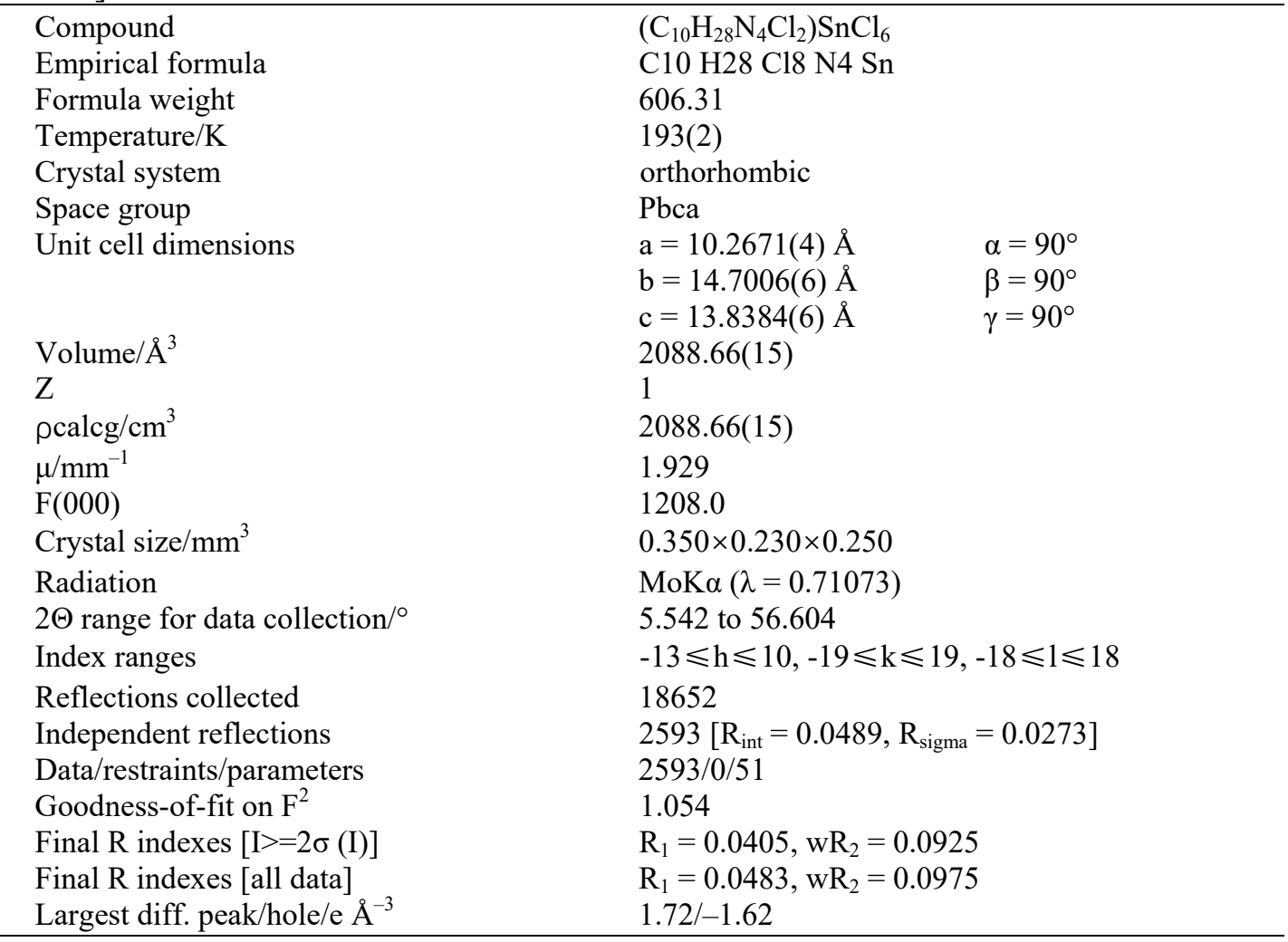



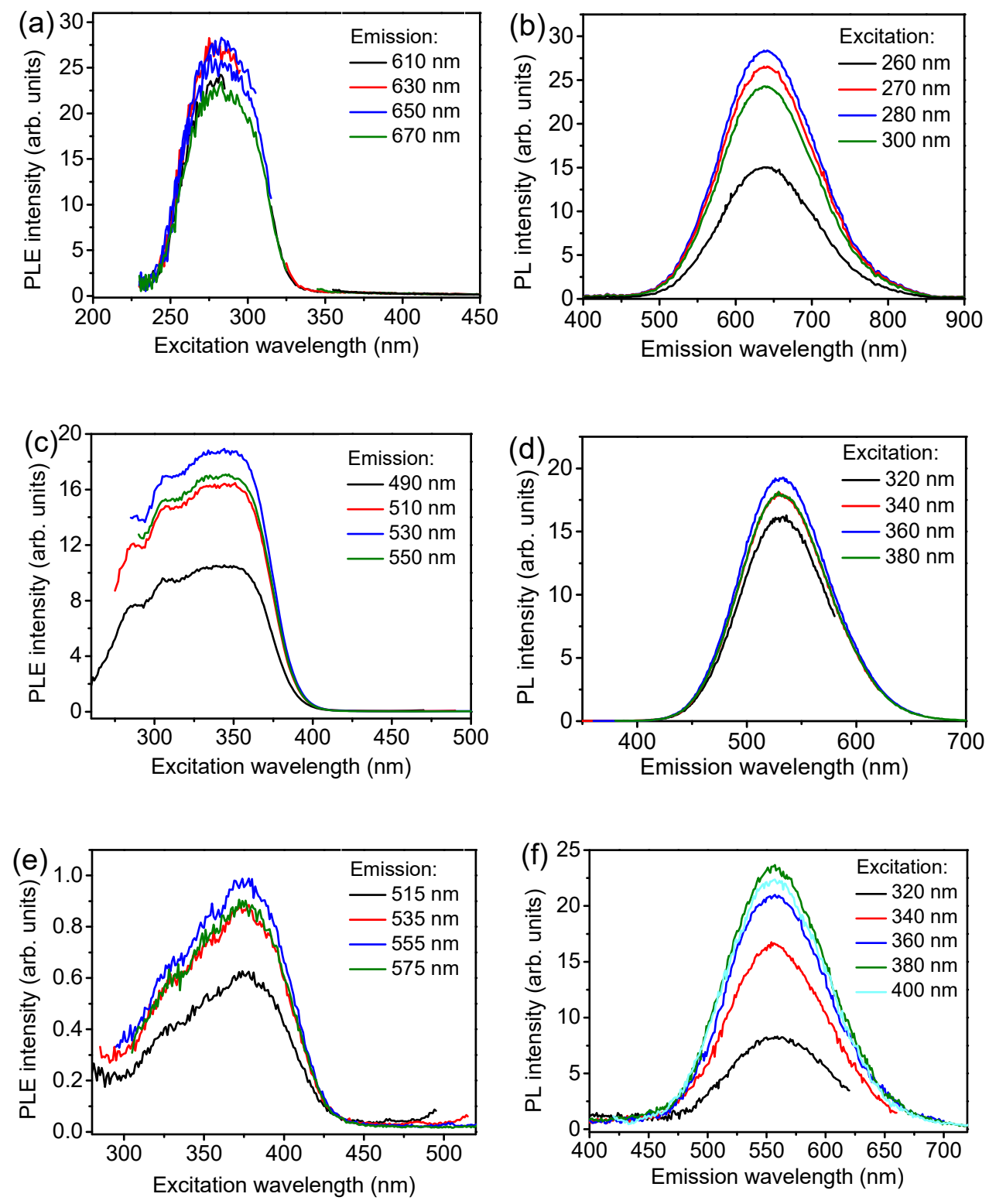

Figure S4. PLE spectra and PL spectra of (a, b) $\left(\mathrm{C}_{10} \mathrm{H}_{28} \mathrm{~N}_{4} \mathrm{Cl}_{2}\right) \mathrm{SnCl}_{4} \cdot 2 \mathrm{H}_{2} \mathrm{O}$, (c, d) $\left(\mathrm{C}_{10} \mathrm{H}_{28} \mathrm{~N}_{4}\right) \mathrm{SnBr}_{6} \cdot 4 \mathrm{H}_{2} \mathrm{O}$, and (e, f) $\left(\mathrm{C}_{10} \mathrm{H}_{28} \mathrm{~N}_{4}\right) \mathrm{SnI}_{6} \cdot 4 \mathrm{H}_{2} \mathrm{O}$ single crystal powders recorded under denoted emission or excitation wavelengths. 

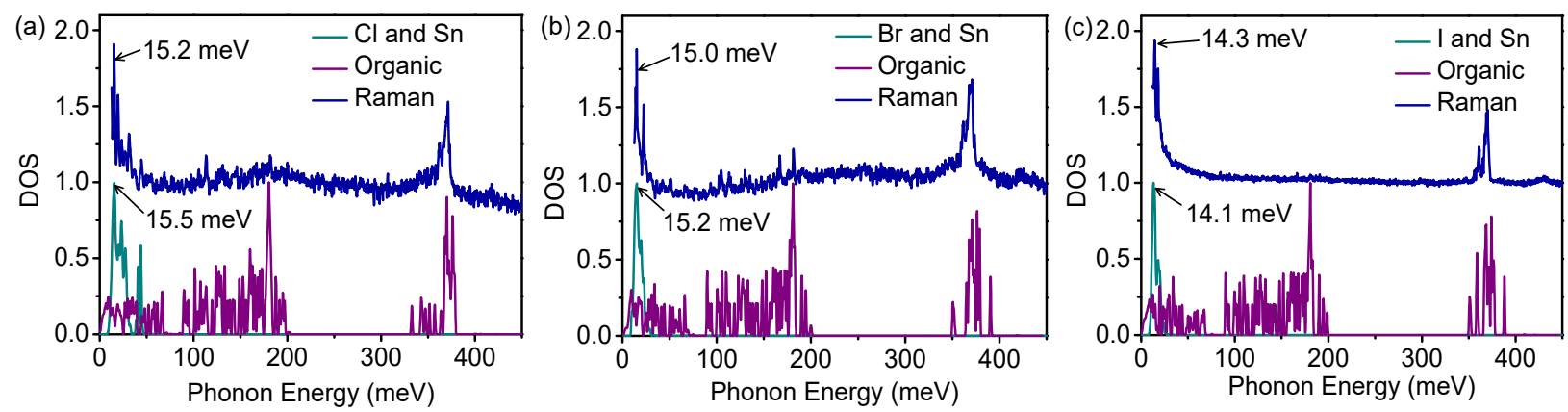

Figure S5. Comparison of calculated phonon DOS and measured Raman spectra of (a) $\left(\mathrm{C}_{10} \mathrm{H}_{28} \mathrm{~N}_{4} \mathrm{Cl}_{2}\right) \mathrm{SnCl}_{4} \cdot 2 \mathrm{H}_{2} \mathrm{O}$, (b) $\left(\mathrm{C}_{10} \mathrm{H}_{28} \mathrm{~N}_{4}\right) \mathrm{SnBr}_{6} \cdot 4 \mathrm{H}_{2} \mathrm{O}$, and (c) $\left(\mathrm{C}_{10} \mathrm{H}_{28} \mathrm{~N}_{4}\right) \mathrm{SnI}_{6} \cdot 4 \mathrm{H}_{2} \mathrm{O}$ single crystals.

Table S10. Calculated ground state $\left(E_{g}\right)$ and first singlet excited state $\left(E_{\text {sin }}\right)$ total energies at the ground state configuration, the lowest triplet state $\left(\mathrm{E}_{\text {trip }}\right)$ and ground state $\left(\mathrm{E}_{\mathrm{g}, \text { trip }}\right)$ total energies at the lowest triplet state configuration, as well as absorption $\left(E_{e x}=E_{s i n}-E_{g}\right)$ and emission $\left(E_{e m}=E_{\text {trip }}-E_{g, t r i p}\right)$ band gaps of the three compounds.

\begin{tabular}{lccccccc}
\hline Compound & $\begin{array}{c}\mathrm{E}_{\mathrm{g}} \\
(\mathrm{eV})\end{array}$ & $\begin{array}{c}\mathrm{E}_{\text {sin }} \\
(\mathrm{eV})\end{array}$ & $\begin{array}{c}\mathrm{E}_{\text {trip }} \\
(\mathrm{eV})\end{array}$ & $\begin{array}{c}\mathrm{E}_{\mathrm{g}, \text { trip }} \\
(\mathrm{eV})\end{array}$ & $\begin{array}{c}\mathrm{E}_{\text {ex }} \\
(\mathrm{eV})\end{array}$ & $\begin{array}{c}\mathrm{E}_{\text {em }} \\
(\mathrm{eV})\end{array}$ & $\begin{array}{c}\mathrm{E}_{\text {ex }}-\mathrm{E}_{\text {em }} \\
(\mathrm{eV})\end{array}$ \\
\hline$\left(\mathrm{C}_{10} \mathrm{H}_{28} \mathrm{~N}_{4} \mathrm{Cl}_{2}\right) \mathrm{SnCl}_{4} \cdot 2 \mathrm{H}_{2} \mathrm{O}$ & -562.93 & -558.80 & -559.36 & -562.64 & 4.13 & 3.28 & 0.85 \\
$\left(\mathrm{C}_{10} \mathrm{H}_{28} \mathrm{~N}_{4}\right) \mathrm{SnBr}_{6} \cdot 4 \mathrm{H}_{2} \mathrm{O}$ & -307.51 & -303.88 & -304.22 & -307.04 & 3.63 & 2.82 & 0.81 \\
$\left(\mathrm{C}_{10} \mathrm{H}_{28} \mathrm{~N}_{4}\right) \mathrm{SnI}_{6} \cdot 4 \mathrm{H}_{2} \mathrm{O}$ & -303.86 & -300.58 & -301.01 & -303.59 & 3.28 & 2.58 & 0.70 \\
\hline
\end{tabular}

Table S11. Quantum yields and average lifetimes of the tin halides along with calculated average radiative and nonradiative lifetimes.

\begin{tabular}{llccl}
\hline Compound & $\mathrm{QY}(\%)$ & $\tau(\mu \mathrm{s})$ & $\tau_{\mathrm{r}}(\mu \mathrm{s})$ & $\tau_{\text {nr }}(\mu \mathrm{s})$ \\
\hline$\left(\mathrm{C}_{10} \mathrm{H}_{28} \mathrm{~N}_{4} \mathrm{Cl}_{2}\right) \mathrm{SnCl}_{4} \cdot 2 \mathrm{H}_{2} \mathrm{O}$ & 92.3 & 9.1 & 9.9 & 118.7 \\
$\left(\mathrm{C}_{10} \mathrm{H}_{28} \mathrm{~N}_{4}\right) \mathrm{SnBr}_{6} \cdot 4 \mathrm{H}_{2} \mathrm{O}$ & 61.7 & 5.3 & 8.7 & 13.9 \\
$\left(\mathrm{C}_{10} \mathrm{H}_{28} \mathrm{~N}_{4}\right) \mathrm{SnI}_{6} \cdot 4 \mathrm{H}_{2} \mathrm{O}$ & 10.8 & 3.7 & 34.2 & 4.2 \\
\hline
\end{tabular}



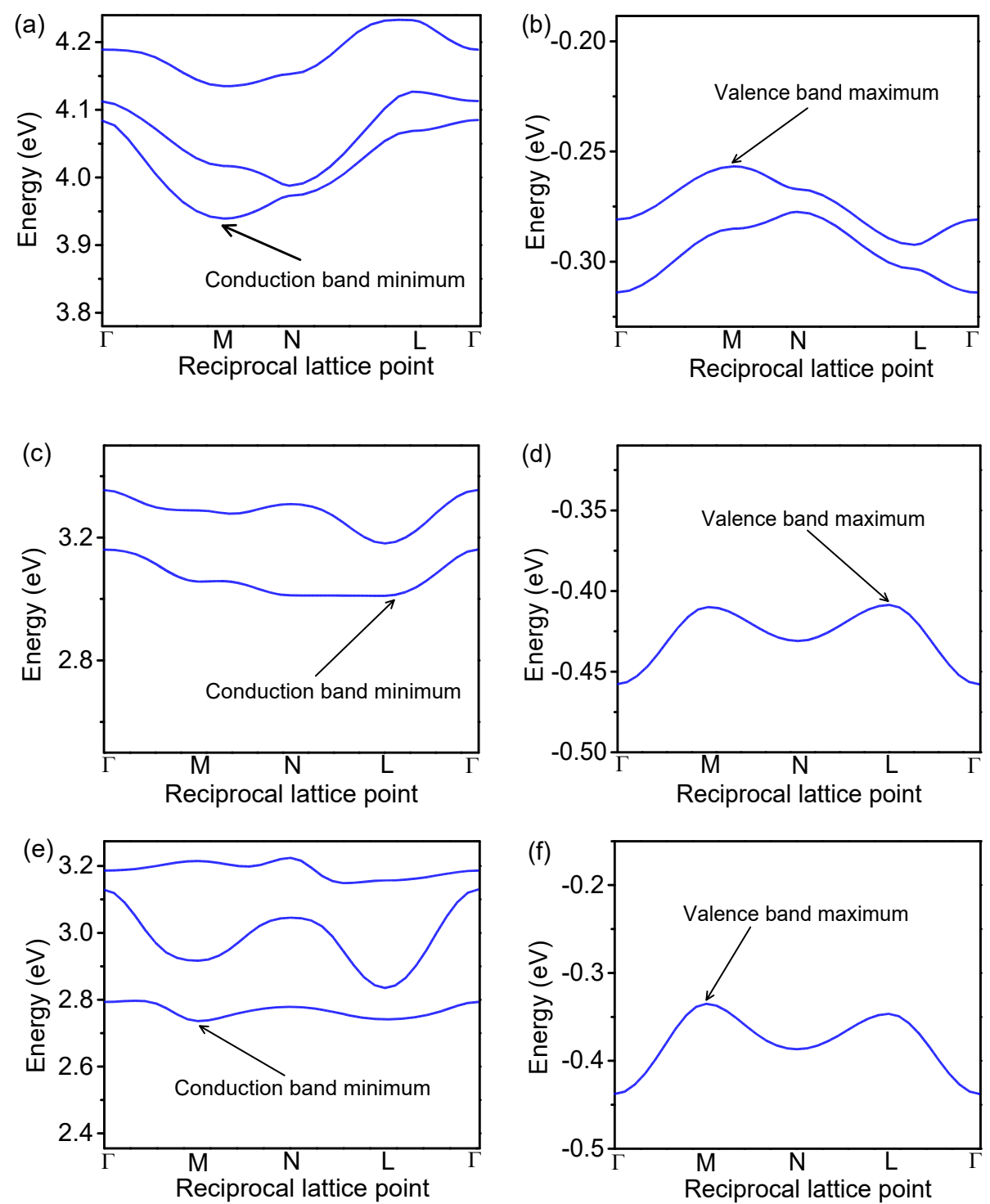

Figure S6. Enlarged areas at the proximity of the conduction band minima and valence band maxima of the electronic structures for $(\mathrm{a}, \mathrm{b})\left(\mathrm{C}_{10} \mathrm{H}_{28} \mathrm{~N}_{4} \mathrm{Cl}_{2}\right) \mathrm{SnCl}_{4} \cdot 2 \mathrm{H}_{2} \mathrm{O}$, (c, d) $\left(\mathrm{C}_{10} \mathrm{H}_{28} \mathrm{~N}_{4}\right) \mathrm{SnBr}_{6} \cdot 4 \mathrm{H}_{2} \mathrm{O}$, and (e, f) $\left(\mathrm{C}_{10} \mathrm{H}_{28} \mathrm{~N}_{4}\right) \mathrm{SnI}_{6} \cdot 4 \mathrm{H}_{2} \mathrm{O}$. 

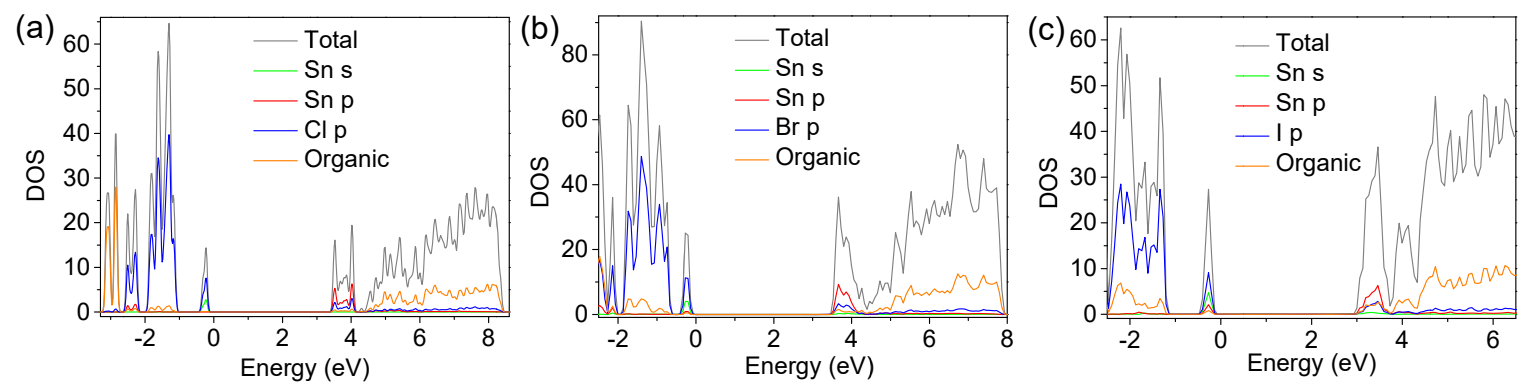

Figure S7. Calculated atom-projected electronic DOS of (a) $\left(\mathrm{C}_{10} \mathrm{H}_{28} \mathrm{~N}_{4}\right) \mathrm{SnCl}_{6} \cdot 4 \mathrm{H}_{2} \mathrm{O}$, (b) $\left(\mathrm{C}_{10} \mathrm{H}_{28} \mathrm{~N}_{4} \mathrm{Br}_{2}\right) \mathrm{SnBr}_{4} \cdot 2 \mathrm{H}_{2} \mathrm{O}$, and (c) $\left(\mathrm{C}_{10} \mathrm{H}_{28} \mathrm{~N}_{4} \mathrm{I}_{2}\right) \mathrm{SnI}_{4} \cdot 2 \mathrm{H}_{2} \mathrm{O}$.
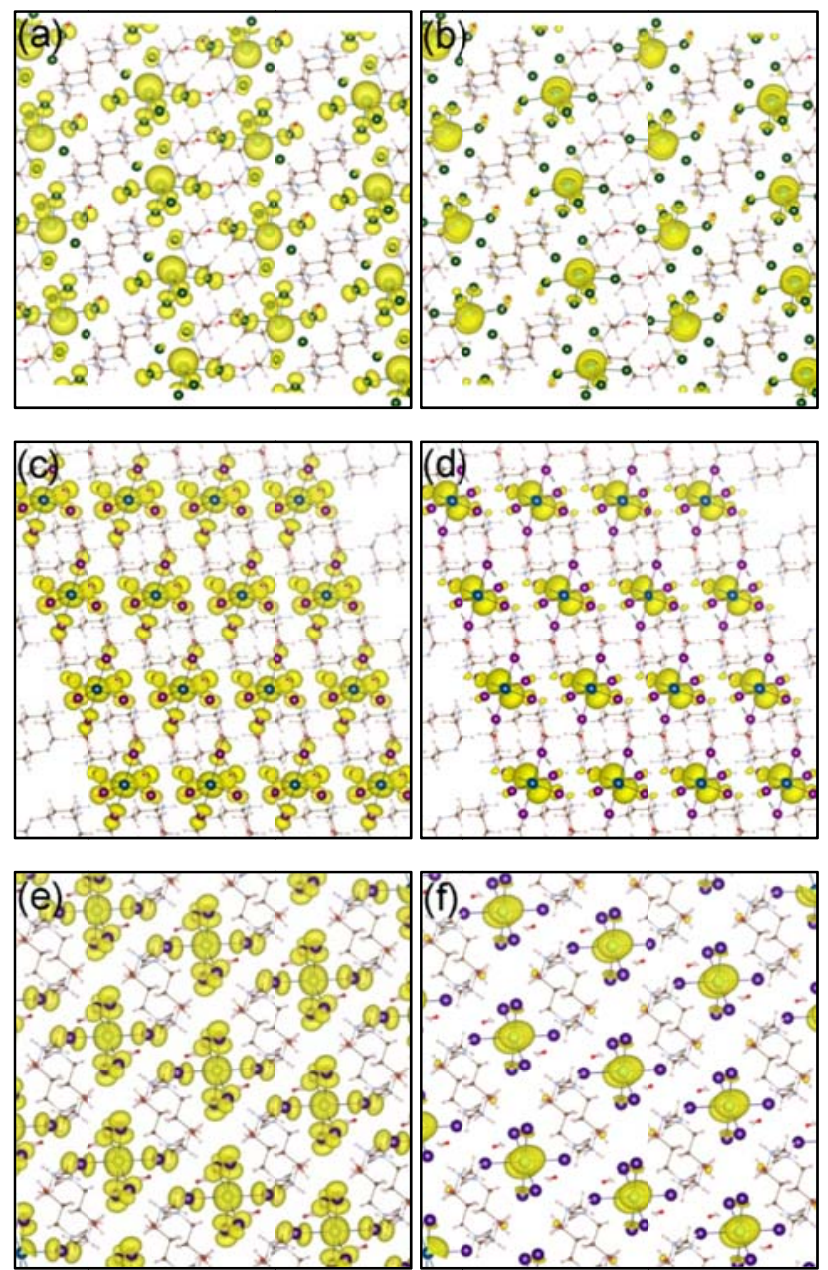

Figure S8. Complete isosurfaces of HOMO and LUMO of (a, b) $\left(\mathrm{C}_{10} \mathrm{H}_{28} \mathrm{~N}_{4} \mathrm{Cl}_{2}\right) \mathrm{SnCl}_{4} \cdot 2 \mathrm{H}_{2} \mathrm{O}$, (c, d) $\left(\mathrm{C}_{10} \mathrm{H}_{28} \mathrm{~N}_{4}\right) \mathrm{SnBr}_{6} \cdot 4 \mathrm{H}_{2} \mathrm{O}$, and (e, f) $\left(\mathrm{C}_{10} \mathrm{H}_{28} \mathrm{~N}_{4}\right) \mathrm{SnI}_{6} \cdot 4 \mathrm{H}_{2} \mathrm{O}$. 


\section{REFERENCES}

(1) Sheldrick, G. M. Crystal Structure Refinement with SHELXL. Acta Cryst. C 2015, 71, 3-8.

(2) Dolomanov, O. V.; Bourhis, L. J.; Gildea, R. J.; Howard, J. A. K.; Puschmann, H. OLEX2: A Complete Structure Solution, Refinement and Analysis Program. J. Appl. Crystallogr. 2009, 42, 339-341.

(3) Kresse, G.; Furthmüller, J. Efficiency of Ab-initio Total Energy Calculations for Metals and Semiconductors Using a Plane-Wave Basis Set. Comput. Mater. Sci. 1996, $6,15-50$.

(4) Blöchl, P. E. Projector Augmented-Wave Method. Phys. Rev. B: Condens. Matter Mater. Phys. 1994, 50, 17953-17979.

(5) Perdew, J. P.; Burke, K.; Ernzerhof, M. Generalized Gradient Approximation Made Simple. Phys. Rev. Lett. 1996, 77, 3865-3868.

(6) Perdew, J. P.; Emzerhof, M.; Burke, K. Rationale for Mixing Exact Exchange with Density Functional Approximations. J. Chem. Phys. 1996, 105, 9982-9985.

(7) Momma, K.; Izumi, F. VESTA: A Three-Dimensional Visualization System for Electronic and Structural Analysis. J. Appl. Crystallogr. 2008, 41, 653-658.

(8) Togo, A; Tanaka, I. First Principles Phonon Calculations in Materials Science, Scripta Mater. 2015, 108, 1-5. 